\title{
Response specificity of male moths to multicomponent pheromones
}

\author{
C.E.Linn, Jr and W.L.Roelofs \\ Department of Entomology, New York State Agricultural Experiment Station, \\ Comell University, Geneva, NY 14456, USA
}

\begin{abstract}
Research on numerous species of moths (Lepidoptera) has shown that the sex pheromones released by females for the purpose of attracting males to mate are multicomponent blends of chemicals. These pheromones serve as species-specific signals, and a major effort in pheromone research has been devoted to determining those factors that influence and control male response specificity. In this paper we review a senes of studies conducted over the past 15 years designed to address the problems associated with measuring the sensitivity of males to blend composition and the influence of individual pheromone components on the active space of the pheromone. Emphasis is placed not only on what has been learned, but also on the changes in methodology that have occurred with advances in analytical procedures, and the importance of a close dialogue between those involved in chemical and behavioral studies.
\end{abstract}

\section{Introduction}

In numerous species of insects successful mate location, courtship and copulation are dependent on a chemical communication channel (Cardé and Baker, 1984). One group in particular, the moths (Lepidoptera), has been studied intensively over the past 25 years, with at least partial pheromone identifications existing for over 300 species (Tamaki, 1985). In most cases the sex pheromone is a female-produced blend of compounds, with males responding to the signal by flying upwind along the odor plume to the female. The upwind-flight response of males to these odor mixtures has been the subject of intense research efforts for several years, and much is known about both the orientation mechanisms involved in the flight response (see Baker, 1989), as well as the sensitivity and specificity of males to the compounds that comprise the pheromone. It is with this latter area that the present review is concerned.

One of the fundamental paradigms in pheromone research relates to the concept of signal specificity, i.e. that sex pheromones function as species-specific mate recognition signals (Cardé and Baker, 1984; Baker, 1985). The primary way in which specificity has evolved in the moths is through the use of mixtures of chemicals, these mixtures differing along taxonomic lines both in their composition as well as the proportion of compounds present. Research on the biosynthesis of sex pheromones has shown that within closely related taxonomic groups (family or sub-family) species often utilize the same or similar compounds in their pheromones, as a result of possessing a common biosynthetic pathway (see Bjostad, 1989). A survey of the moths thus shows that across families or sub-families, different sets of compounds are utilized (varying in chain length, position of double bonds or functional group), whereas within these taxonomic groups closely related species utilize unique ratios of common components. By varying the qualitative properties of the signal along these two dimensions an enormous variety of mixtures have evolved, providing each species with a unique communication signal.

The concept of signal specificity has been a central force in the analysis of behaviors exhibited by male moths to their conspecific pheromone. Clearly, male response is 
dependent on detection and recognition of a precise mixture of compounds. The present paper will review the research effort of this laboratory in the area of signal and response specificity over the past 15 years (reference to 'our' in the following discussion refers to studies conducted by personnel connected directly with the laboratory of W.L. Roelofs). During this period dramatic changes have occurred in our understanding of how males perceive and respond to these complex odor mixtures. The review is not intended to cover the entire body of literature in this field, but rather will focus on the problem of measuring the sensitivity of male moths to odor quality, and the role

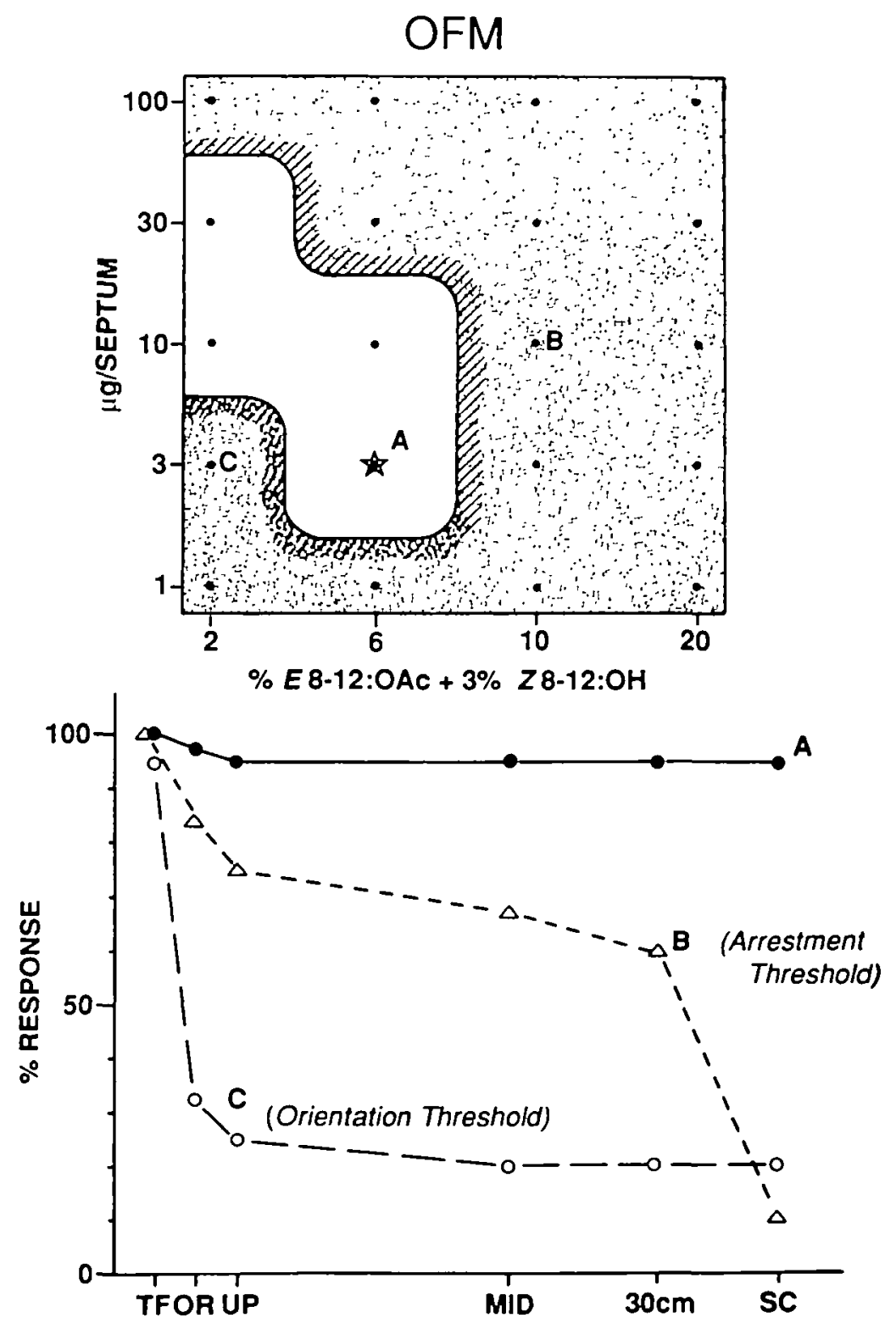


of individual compounds in influencing various phases of the long-distance upwind flight response of males. In addition to presenting a summary of what we have learned, emphasis will be placed on the development of concepts and methodologies that have influenced the evolution of our thinking, and in particular will stress the importance of an active dialogue between those involved in chemical, biochemical and behavioral investigations.

\section{Male sensitivity to odor quality}

One example of a group in which the concept of response specificity is well illustrated is found within the tortricid moths that feed on apple trees (Roelofs and Brown, 1982). A number of species utilize different ratios of $(Z)$ - and $(E)$-11-tetradecenyl acetate (Z/E11-14:OAc) in their pheromones, and although these species are sympatric and synchronic in their habits, in field trapping experiments they exhibit a high degree of specificity for their particular ratio of iosmers, with very little cross-attraction between closely related species. Beginning in the 1970 s this pattern of acute sensitivity to the $\mathrm{Z} / \mathrm{E}$ isomer ratio emerged as a general principle across many familes of moths and subsequently became the focus of the first attempts to understand the response specificity of male moths for their conspecific pheromones.

\section{The threshold hypothesis}

In 1979 Roelofs proposed the threshold hypothesis for moth perception of multicomponent pheromones (Roelofs, 1978). According to the hypothesis male response specificity is controlled by two major 'threshold' effects on flight behavior that are related directly to male perception of (i) the ratio of components and (ii) the release rate of pheromone. The hypothesis originally proposed that the two effects were associated with lower or higher doses of a given ratio of components. The lower dose threshold was thought to affect the initiation of upwind flight in males, an early step in the flight sequence, while the higher dose threshold was thought to affect males later in the flight sequence, after they had initiated upwind flight.

Our tests of the threshold hypothesis have largely involved the Oriental fruit moth, Grapholita molesta (Busck). The pheromone of this species is a three component mixture

Fig. 1. A summary from flight tunnel studies on the response specificity of male Oriental fruit moths to their three-component sex pheromone. The top shows the matrix of $20 \mathrm{Z} / \mathrm{E}$ isomer-dose combinations tested, with each treatment containing $3 \%$ of the $\mathrm{Z} 8-12: \mathrm{OH}$ component. This is one of five sets, the others contanning $0,10,30$ and $100 \% \mathrm{Z8}-12: \mathrm{OH}$. The 'area of peak attraction' is the region enclosed by the contour line, drawn here in a highly diagrammatic manner. The region includes those four blend-dose combinations that elicited high levels of upwind flights and source contacts, levels not significantly different from the maxımal response, designated by the star (A). Sub-optımal blend-dose combinations adjacent to the area of peak attraction are those that elicit behavior in males that can be characterized by one of two threshold effects. Two blend-dose combinations, designated at $B$ and $C$, illustrate the two threshold effects on male flight behavior, and the response of males in the flight tunnel to these two combinations, as well as to the one eliciting maximal response (at A), are shown in the bottom of the figure. The arrestment effect (B, representative of treatments containing higher doses or higher $\$ \mathrm{E}$ ratios from those within the peak area) occurs primarily near the completion of the upwind flight, whereas the effect on orientation (C, representative of lower doses and lower $\% E$ blends) to the odor plume occurs in the early phase of the response, prior to initiation of upwind flight. Behavors are taking flight (TF), orientation in the odor plume (OR), initiation of upwind flight (UP), upwind flight to the mid-point (MID) and to within $30 \mathrm{~cm}$ of the source, ending with source contact (SC). Taking flight occurs $\sim 150 \mathrm{~cm}$ downwind of the source. Taken from Linn and Roelofs (1983). 
composed of (Z)-8-dodeceynl acetate (Z8-12:OAc), with 6\% E8-12:OAc and 3-30\% Z8-12:OH (Cardé et al., 1979). In an extensive series of flight tunnel tests (see Baker et al., 1981; Linn and Roelofs, 1983) males were exposed individually to one of a range of doses of the Z/E isomer mixture containing increasing amounts of the Z8-12:OH component (see Figure 1). In the flight tunnel males were scored for each behavior performed in the sequence: wing-fanning activation response, leading to flight from their release cage, orientation in an upwind direction in the odor plume, upwind flight to the source (typically over a 1-3-m distance), where a courtship display or copulatory attempt may be made (Baker and Linn, 1984). In this way changes in male behavior at a particular step in the sequence could be correlated with specific changes in the pheromone blend. Parallel field trapping studies were also conducted using the same sets of treatments.

The results of these studies demonstrated three important principles concerning male moth perception of odor mixtures. First, male response was dependent on the presence of the three components. Completed flights to the source did not occur to any partial combination of components. Second, males exhibited maximal levels of upwind flight and source contacts to a narrow range of doses of the natural $6 \% \mathrm{E}$ mix, the doses centered around that which best approximates the release rate of females. Third, male flight behavior was differentially affected by changes in the proportion of the two minor components. Maximal, or peak, levels of response dropped significantly with slight changes in the $\mathrm{Z} / \mathrm{E}$ ratio of iosmers, indicating a high degree of sensitivity to this parameter of the signal, whereas males were less sensitive to varying amounts of the Z8-12:OH component, with peak levels of source contacts, and attraction in the field, occurring to blends containing $3-100 \%$ of this component added to the $\mathrm{Z} / \mathrm{E}$ mixture.

The analysis also confirmed that male response specificity was controlled by two distinct effects on male in-flight behavior, as proposed in the threshold hypothesis (see Figure 1). It should be noted, however, that our present interpretation of these thresholds is not the same as originally proposed. The term threshold usually refers to the lowest stimulus level that produces a behavior in an individual, or can be detected by a sensory organ (Levine and Shefner, 1981), and this was the general intent in the original presentation of the hypothesis. Alternatively, one can focus on the response to an optimal, or peak, stimulus and ask the question: how variable can this stimulus be before a significant decrease in effectiveness occurs? This would then constitute a different type of 'threshold' stimulus. Our data showed that the female-produced blend represents a stimulus that elicits maximal response, a stimulus to which males are presumably 'tuned'. By analyzing the response profiles for each dose-blend combination (how many insects completed each of the steps in the flight tunnel sequence) we were then able to determine the degree of variability in the qualitative and quantitative parameters of the signal that can be allowed without having a significant decrease in peak levels of response. In a plot of pheromone dose versus the ratio of components, we characterized this range of variability as the 'area of peak attraction' (see Figure 1).

It is the blend-dose combinations at the boundary of this area that we were most interested in, because it is with these signals that the two proposed 'threshold' effects are observed. As noted above our data showed that these effects were easily characterized, clearly associated with specific blend-dose combinations, and resulted in highly significant reductions in the proportion of males making contact with the source. 
The first threshold effect was characterized as a flight 'orientation threshold'. Blend-dose combinations eliciting this effect were predominantly at lower doses and ones containing lower \% $\mathrm{E}$ ratios compared to the dose of the natural $6 \% \mathrm{E}$ blend that elicited maximal response levels. Males typically took flight in high proportions to these treatments but subsequently failed to orient in the plume and initiate upwind flight. The second was characterized as an 'arrestment threshold'. Blend-dose combinations eliciting this effect were predominantly at higher doses and ones containing higher $\not{F} \mathrm{E}$ ratios than that released by females. Males would typically take flight and initiate upwind-oriented flight in high numbers, but then would abruptly halt their upwind flight progress, hover in a stationary position for a brief period and then fly vertically out of the odor space [see Baker (1985) for a discussion of the behavioral and neurophysiological basis for the arrestment effect]. The results of parallel field tests using the same series of blend-dose combinations supported the conclusions concerning male response specificity, with males exhibiting a high degree of selectivity for the natural female-produced blend of compounds (Baker et al., 1981; Linn and Roelofs, 1983).

\section{A comparative study with the pink bollworm moth}

Our studies with the Oriental fruit moth showed that males of this species possess a remarkable degree of sensitivity to odor quality and confirmed the hypothesis that specific changes in blend quality can result in distinct effects on male upwind flight behavior. The question remained, however, as to the generality of these findings, and to assess this a comparative study was conducted using the pink bollworm moth Pectinophora gossypiella (Saunders). The sex pheromone of this species is a two-component mixture (61:39) of $(Z, Z)$ - and $(Z, E)-7,11$-hexadecadienyl acetates (Haynes et al., 1984). This species was chosen, in part, because field studies had suggested that males responded to a wider range of component ratios than was observed with the Oriental fruit moth (see Roelofs, 1978). We conducted an extensive flight tunnel study in a manner similar to that with the Oriental fruit moth (Linn and Roelofs, 1985) and found that our results were considerably different than expected, based on the previous studies. Male pink bollworm moths exhibited a much lower degree of response specificity, in that they were responsive in peak numbers to a much wider range of component ratios and release rates than male Oriental fruit moths. Further, the boundary of the area of peak attraction was not as distinct; rather, response levels fell gradually with distance from the central blend - dose combination eliciting maximal response. Finally, lowered response to sub-optimal combinations was the result of the threshold effect on orientation to the odor plume, with no evidence for an arrestment effect at higher doses or higher ZZ/ZE isomer ratios.

The conflicting results were first hypothesized to be due to differences in selection pressure for a narrowly tuned signal. Presumably, one of the factors that has led to the evolution of complex odor mixtures is the requirement for a distinctive signal in an environment where other species using the same or similar compounds might be present (Cardé and Baker, 1984). Different species might then show varying degrees of specificity depending on the degree of overlap or contact with closely related forms.

While this is a plausible hypothesis in its own right, the discrepancy between the results obtained with the Oriental fruit and the pink bollworm moths was resolved in 
a subsequent study that illustrated the impact that temperature can have on male detection of these signals (Linn et al., 1988a). The original study with Oriental fruit moth was conducted at $20^{\circ} \mathrm{C}$, whereas that with the pink bollworm moth was at $26^{\circ} \mathrm{C}$, based on published information from previous flight tunnel studies with each species (Farkas et al., 1974; Baker and Cardé, 1979). Serendipitously during other studies with the Oriental fruit moth, we observed that males of this species, when tested at $26^{\circ} \mathrm{C}$, exhibited significantly higher levels of upwind flight to higher than normal $\%$ E8-12:OAc ratios (e.g. 20 versus $6 \%$ ), and a preliminary study confirmed that temperature could significantly alter male sensitivity to the ratio of components. We thus decided to conduct a new flight tunnel study with both species in which males were tested with a range of blend-dose combinations at each of the two temperatures. Our results showed that indeed the area of peak attraction for both species could be significantly altered by acclimating and testing males at the different temperatures (Linn et al., 1988a). At each temperature, the blend-dose combination eliciting maximal response remained the same, even though the range of combinations eliciting similar levels of response was significantly greater at the higher temperature. Thus, the area of peak attraction was expanded with higher temperature, rather than simply shifted, suggesting that the change was due to an alteration in male perception of the signal rather than to an increase in the release rate of the pheromone. Equally important was the fact that at the lower temperature both threshold effects on male flight behavior were observed, whereas at the higher temperature the arrestment effect was significantly diminished.

With respect to response specificity in the field, the significance of the observed temperature effect on male perception of pheromone is difficult to assess. This is because of the complex relationship that exists between temperature and the diel periodicity of the response (Cardé and Webster, 1981). A number of studies have shown that changes in temperature that occur during a particular time of the photoperiod can shift the daily period of mating activity, so that it occurs at the most favorable time. Thus, the degree of specificity is probably linked to a most favorable temperature, one at which the insects can be active by advancing or delaying the time at which female release of pheromone and male response occurs. While at the present time this remains an open question, the temperature effect on male response specificity has been studied at the level of the antennal receptors, and is clearly associated with differential rates of adaptation to individual pheromone components (see Baker et al., 1989).

\section{Summary}

The threshold hypothesis has provided a good working model to explain male response specificity. The studies with Oriental fruit and the pink bollworm moths showed that male moths can exhibit remarkable degrees of sensitivity to the qualitative properties of their pheromone and that this specificity is controlled by two main effects on male behavior, the orientation and arrestment thresholds. The two threshold effects have also been documented in a number of other moth species (see Glover et al., 1988; Linn et al., 1984, 1985), and both the arrestment effect as well as the temperature-induced changes in moth perception are being successfully studied at the level of the nervous system (see Baker, 1985; Baker et al., 1989). Our studies also demonstrate, however, that the degree of specificity is not fixed, but rather can display considerable plasticity. This is evidenced by the above-discussed temperature effect, as well as by earlier studies 
in which the area of peak attraction in Oriental fruit moth was considerably expanded by pre-exposing individual males to the E8-12:OAc component of the pheromone (see Linn and Roelofs, 1981). Thus, while males of a given species may be genetically 'tuned' to a particular signal, their specificity for that signal is subject to environmental influences as well as experience.

\section{Male sensitivity to odor composition: what are pheromone components and what do they do?}

The studies discussed above on male moth sensitivity to odor quality illustrate the importance of blend composition and ratio in effecting peak levels of response. Clearly, the success of these investigations was dependent on an accurate and complete characterization of the chemicals in the pheromone and their ratios as produced or released by females. While this objective is clear enough conceptually, a fundamental problem in the analysis of sex pheromones relates to the question of what a pheromone component is. How do we know that a compound, or mixture of compounds, is part of the pheromone, and how do we know that what we have isolated comprises the entire complement of chemicals? Historically, pheromone identifications have been plagued by two major problems: (i) being able to isolate analytically the very small amounts of components present in the female gland; and (ii) the lack of information on how these signals are produced by females and, therefore, a method for predicting whether a compound might or might not be part of the pheromone. In turn these problems have significantly affected the way in which male behavior has been studied.

\section{Pheromone components, behavioral functions and a behavioral paradigm}

The majority of multicomponent sex pheromones are composed of a single abundant compound (commonly referred to as the 'major' component) and several others present at much lower levels ('minor' components, usually $<10 \%$ of the amount of the major component). Historically a typical pheromone study initially involved chemical identification of the most abundant compound. This was primarily because analytical procedures, e.g. gas chromatography, were not sensitive enough to detect the presence of less abundant compounds (it should be remembered that quantitatively most moth pheromones are in the range of micograms to nanograms/female), but also because there was no a priori reason for expecting any additional components to be present (see Bjostad, 1989).

Following a chemical identification of the major component, it was then tested for behavioral activity. This usually involved a laboratory wing-fanning bioassay or a field trapping experiment. Additional compounds were added to the pheromone, either as a result of more extensive chemical analysis, or more often on the basis of field trials. In these field trials, a series of doses and ratios of components was evaluated, the components many times being candidates based simply on their similarity to the major component or their activity with other species. The potency of pheromone traps as indicators of male behavioral activity proved to have a significant impact on these 'one-compound-at-a-time' pheromone identifications.

It is important to note that during this period of study (predominantly in the 1970s), there was no a priori basis for accepting or rejecting a component as part of the 
pheromone. It was thus necessary to establish the criterion that for a compound to be considered part of the pheromone it must, when added to the existing pheromone, enhance the level of response over that observed previously (see Cardé, 1979). It was assumed (and logically so) that if male response was enhanced by some compound when present in a certain proportion of the mixture, then this mixture must be close to the natural pheromone.

Using this experimental protocol it was observed that in many cases the enhanced level of response was the result of the added compound triggering a specific behavior in the male flight sequence, e.g. landing at the source or the courtship display. These results led to the development of a paradigm (hereafter referred to as the 'component' paradigm) describing the male response, from far downwind to courtship display and copulation, as a sequence of steps, with each step dependent on the presence of additional components in the blend (Cardé et al., 1975a,b; Baker et al., 1976; Roelofs and Cardé, 1977; Linn and Gaston, 1981; Howse et al., 1986; see Cardé and Charlton, 1984). According to this paradigm, major components were responsible for long-range activation and upwind flight by males, and thus for determining the dimensions of the active space of the pheromone, whereas minor components functioned to sustain upwind flight close to the source, or to trigger specific close-range behaviors, e.g. landing and courtship displays.

The 'component' paradigm proved highly successful in explaining the pheromonemediated responses of several insects and became the foundation for future behavioral studies (Roelofs and Cardé, 1977). As will be illustrated below, however, subsequent and more sensitive chemical analysis of several of these chemical communication systems showed that much of the behavioral evidence supporting the component paradigm was collected using pheromone blends that, while proving highly attractive in field trapping experiments, were, in fact, not the blends produced and released by females.

\section{Biosynthetic pathways, pheromone components and behavioral assays: a case study with the cabbage looper}

The imbalance in our weighting of behavioral versus chemical evidence for a given candidate compound changed dramatically as a result of the findings from studies on the biosynthetic pathways by which females produce their pheromone components (Roelofs and Bjostad, 1985; Bjostad, 1989). These studies provided the impetus for a major change in our approach to the analysis of male behavior in the flight tunnel and to a new set of criteria for evaluating the status of a proposed pheromone component (Linn et al., 1984).

The impact of this research can best be illustrated by an example, and in the following discussion we will focus on the communication system of the cabbage looper moth, Trichoplusia ni (Hübner). The major component of this species' pheromone was identified as (Z)-7-dodecenyl acetate (Z7-12:OAc) (Berger, 1966). This compound was utilized for several years in laboratory and field studies and satisfies the criteria of a major pheromone component (see Shorey, 1976). A second component, dodecyl acetate (12:OAc) (Bjostad et al., 1980), was shown to be present at a level of 7\% in the two-component blend. Using a sustained-flight tunnel, Linn and Gaston (1981) showed 
that 12:OAc, while not itself affecting male behavior, did significantly enhance the close-range flight response of males when presented in the appropriate ratio with the major component Z7-12:OAc. The cabbage looper pheromone thus appeared to fit the criteria of the existing behavioral paradigm, functioning as a two-component blend composed of a primary and secondary component, with each affecting male behavior in different ways.

Our interest in the cabbage looper was rekindled as a result of studies on the biosynthetic pathway for this species' pheromone (Bjostad et al., 1985), in which it became apparent that several additional compounds were present and released by females. In fact the female-produced pheromone is a six-component blend composed of Z7-12:OAc, 12:OAc, Z5-12:OAc, 11-12:OAc, Z7-14:OAc and Z9-14:OAc (Bjostad et al., 1985). In approaching the behavioral analysis of this new pheromone blend, two aspects of the biochemical analysis were significant. First, the technological advances in capillary gas chromatography allowed very sensitive profiles to be obtained of gland contents from individual females. These profiles, as well as those from females actually releasing pheromone, were highly consistent with respect to the number and proportions of compounds released. Second, the new compounds had been predicted on the basis of a theoretical framework describing the biosynthetic pathway for the pheromone.

Use of the flight tunnel also proved to be a significant factor in the identification of the new cabbage looper pheromone. Throughout the process males were routinely tested to the synthetic mixture, to calling females, and to extracts, all of which could also be analyzed by capillary GLC providing a valuable method for cross-checking the chemical analysis. The preliminary screening of the synthetic mixture in the flight tunnel showed that very high levels of response (consistently $>90 \%$ source contacts) could be achieved with the six-component blend, and that these levels were the same as observed with female extracts or calling females. In addition, and of significance, was the fact that the response to these three sources was superior to that obtained with the previously identified two-component mix.

These initial findings were of great importance because they not only added to our confidence concerning the accuracy of the identification, but also provided a clue to a major problem that had occurred in the previous study with the two-component blend (Linn and Gaston, 1981). In the previous studies (Linn and Gaston, 1981) the standard against which the effect of 12:OAc was measured was the response to Z7-12:OAc alone. This followed from the assumption that a component must enhance male response when added to a previously identified pheromone in order to be considered part of the chemical signal. However, the response to the major component proved to be highly variable when evaluated on a day-to-day basis. At the time this variability was attributed to problems with the flight-tunnel environment, or to variation in male viability or sensitivity, and not to the possibility that the chemical identification was incomplete. The result was that data for many days was simply not included in the final analysis. Thus, while the observed effect of the minor component was a valid one, the levels of response to the two-component blend versus the major component alone were in fact much lower than reported and, as we now recognize, represent the response of a low level of the population of males to a partial blend of the female-released pheromone. 
Clearly, if we had tested extracts or calling females against the response to the synthetic two-component mix, a very different conclusion could have been reached concerning the action of 12:OAc in the cabbage looper pheromone.

With the completion of the identification of the new components it became apparent that a large behavioral study was warranted, in order to determine the importance of each minor component on the male flight response. The impact of the combined biochemical and flight tunnel tests, however, suggested that a different approach to the behavioral analysis might be more appropriate. That is, rather than looking for enhanced affects of individual components when added to the major component, we began our flight-tunnel tests with the hypothesis that the six-component blend was the cabbage looper pheromone, and that a more appropriate protocol for assessing the importance of each of the new compounds would involve the subtraction of components from the blend. The results of these tests were both surprising and unexpected. Tests with the five-component blends showed that $\mathrm{Z7-12:OAc}$ was the only component that significantly affected male behavior when removed from the blend. In other words, any of the minor components could be removed without affecting peak levels of source contacts. Analysis of the four-component blends showed that only when both of the 14-carbon acetates were removed was there a reduction in peak response levels. The results of the entire subtraction series produced two important findings. First, it was clear that certain minor components could substitute for one another in the blend, and thus that the chemical signal produced and released by females has an element of redundancy in it. Second, it was equally clear in the context of the entire male-response sequence that the individual minor components were not responsible for triggering specific behaviors.

\section{Pheromone components, behavioral functions and a new paradigm}

These results suggested that the component paradigm might be in error, and that greater efforts should be placed on examining the influence of the female-produced blend on male behavior. We now, in fact, recognize that previous studies with the cabbage looper moth, the Oriental fruit moth and a third important species, the redbanded leafroller, Argyrotaenia velutinana (Walker), all of which supported the component paradigm, utilized pheromone blends that are not those released by conspecific females (Cardé et al., 1975a,b; Baker et al., 1976; Linn and Gaston, 1981). In the case of the Oriental fruit moth and the redbanded leafroller this involved partial blends with exaggerated levels of minor components, proposed largely on the basis of field trapping experiments, whereas the cabbage looper tests were done using an incomplete blend.

New chemical and behavioral re-investigations with each species have resulted in a reversal in our opinion concerning the functional role of minor components (Baker and Cardé, 1979; Linn et al., 1984, 1985, 1987; Bjostad et al., 1985). In our flight-tunnel tests, males, when tested over a series of doses, were significantly more responsive to the female-produced blends than to the major components alone strongly suggesting that, far-downwind of the female, males are responding to the complete mixture of components (Figure 2). This further implies that the 'active space' of the pheromone is a function of male perception of the blend rather than the most adundant component, and that even though minor components may be present in low amounts, their presence 


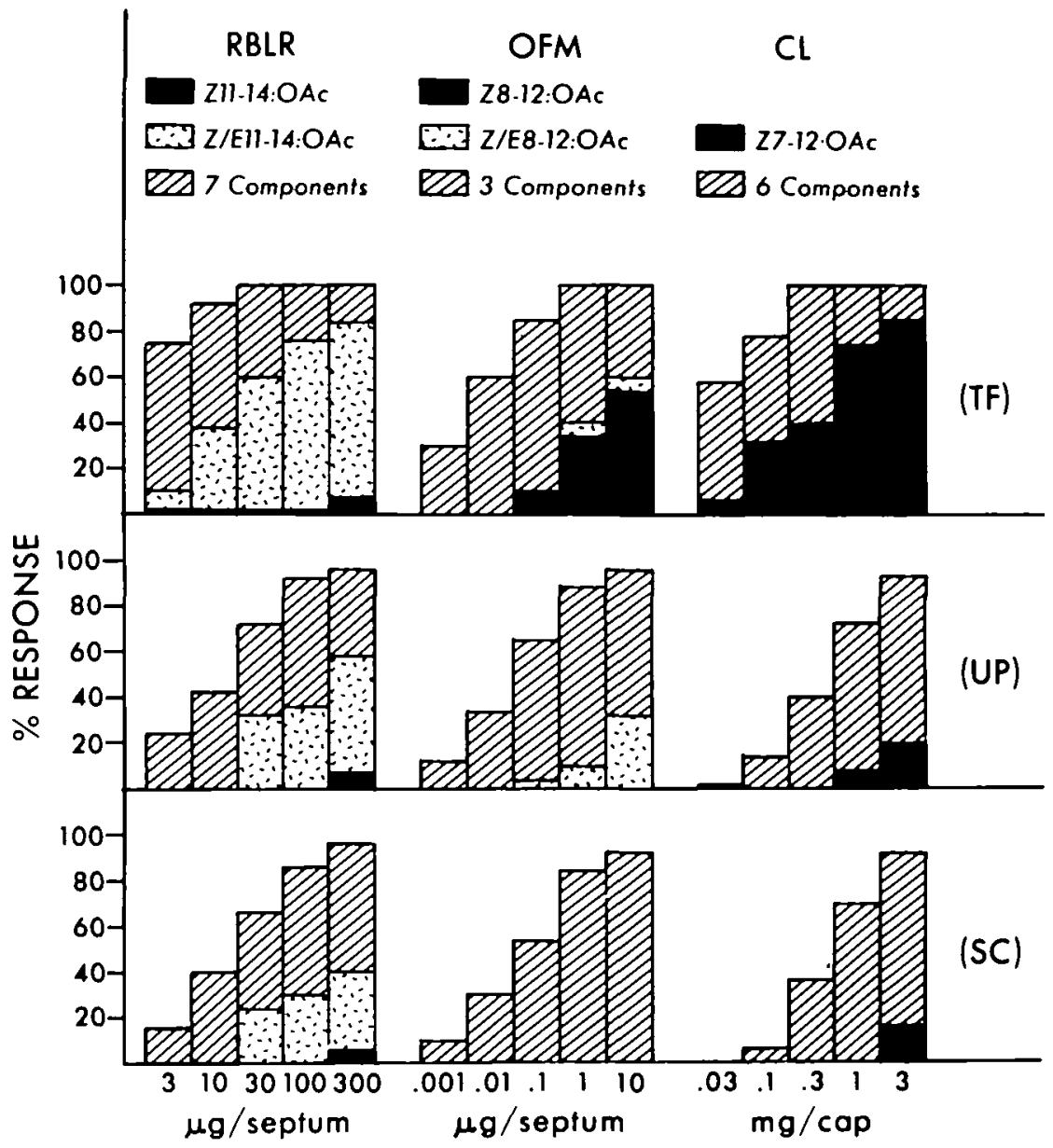

Fig. 2. Percentage of male redbanded leafroller, Oriental fruit moths and cabbage loopers exhibiting three key behaviors in the sustained-flight tunnel (taking flight, TF; upwind flight over a 1.5-m distance, UP; and source contact, SC). Responses for each species are to the major component alone (solid area), the $\mathrm{Z} / \mathrm{E}$ mixture (stippled area) and the complete blends as produced by females (hatched area). Doses represent the amount of the major component applied to the source, with the other components added to achieve the correct proportions. The dose series for each species was selected to include a release rate approximating that of a female, as well as several lower release rates. Data from Linn et al. (1985).

is still necessary to effect male response at distances far downwind of the female. Direct evidence for this in the field came from our most recent studies with the Oriental fruit moth (Linn et al., 1987). We adopted a procedure first developed with this species by Baker and Roelofs (1981), in which the wing-fanning activation response of males was measured in field conditions. Using a stream of visible bubbles to follow the meandering plume, the distance downwind that male Oriental fruit moths wing-fanned could be measured. The results of these studies showed clearly that, with respect to the number of insects responding as well as the distance downwind that they activated, 


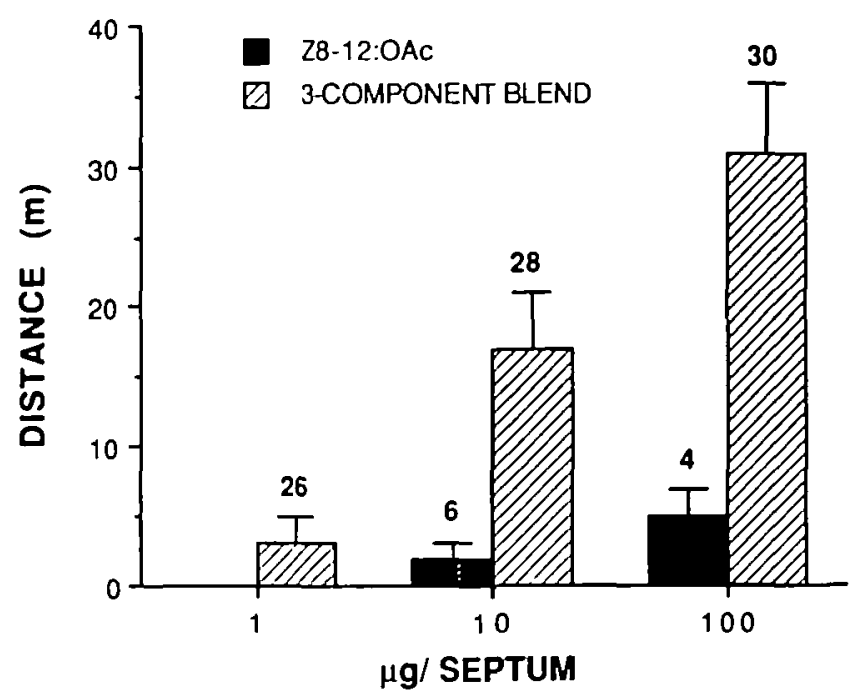

Fig. 3. Distance downwind that prompted male Oriental fruit moths to exhibit a wing-fanning activation response. Three doses of the major component (Z8-12:OAc) or the three-component blend (Z8-12:OAc + 6\% E8-12:OAc $+3 \% \mathrm{Z8}-12: \mathrm{OH})$ were tested. Response values are mean $+\mathrm{SD}, n=30$ for each treatment. Values above each bar are the number of insects of the 30 tested that activated. Data from Linn et al. (1987).

males are far more sensitive to the three-component blend than to the major component alone (Figure 3).

The case with the redbanded leafroller is particularly illustrative of the caution that should be exercised in proposing a particular blend largely on the basis of field trapping experiments. Field tests had shown that the addition of 12:OAc to the previously identified mixture of Z- and E11-14:OAc (8\% E) in a ratio of $1-1.5$ times the level of $\mathrm{Z}$ isomer (the major component) significantly increased trap catch. Observations of male behavior in the laboratory and the field showed further that the $\mathrm{Z} / \mathrm{E}$ isomer mix was responsible for sustained upwind anemotactic flight, while 12:OAc significantly enhanced close-range approach, landing and attempted copulations (Baker et al., 1976). Chemical analysis confirmed that 12:OAc was present in female glands, but a ratio of only 3-5\% of the $Z$ isomer. At this ratio, 12:OAc did not enhance trap catch, and it was concluded on the basis of field trapping studies that females must be rapidly synthesizing and releasing larger amounts of 12:OAc. This illustrates, again, the influence that field studies had over the less-sensitive chemical analyses. Our most recent evidence, however, indicates that 12:OAc is but one of seven pheromone components for the redbanded leafroller and that the above-described behavioral scenario, while correct for the three-component mix, is not correct for the complete female-produced blend (Figure 4).

The reversals obtained with the Oriental fruit moth, cabbage looper and redbanded leafroller moths support a different interpretation of male perception of odor mixtures, that the blend acts as a unique entity, or unit, to effect optimal male behavior. Our present position, based on the combined studies with these three species, is that the female-produced blend is the relevant signal in eliciting male response, including longdistance attraction, courtship and copulation. 


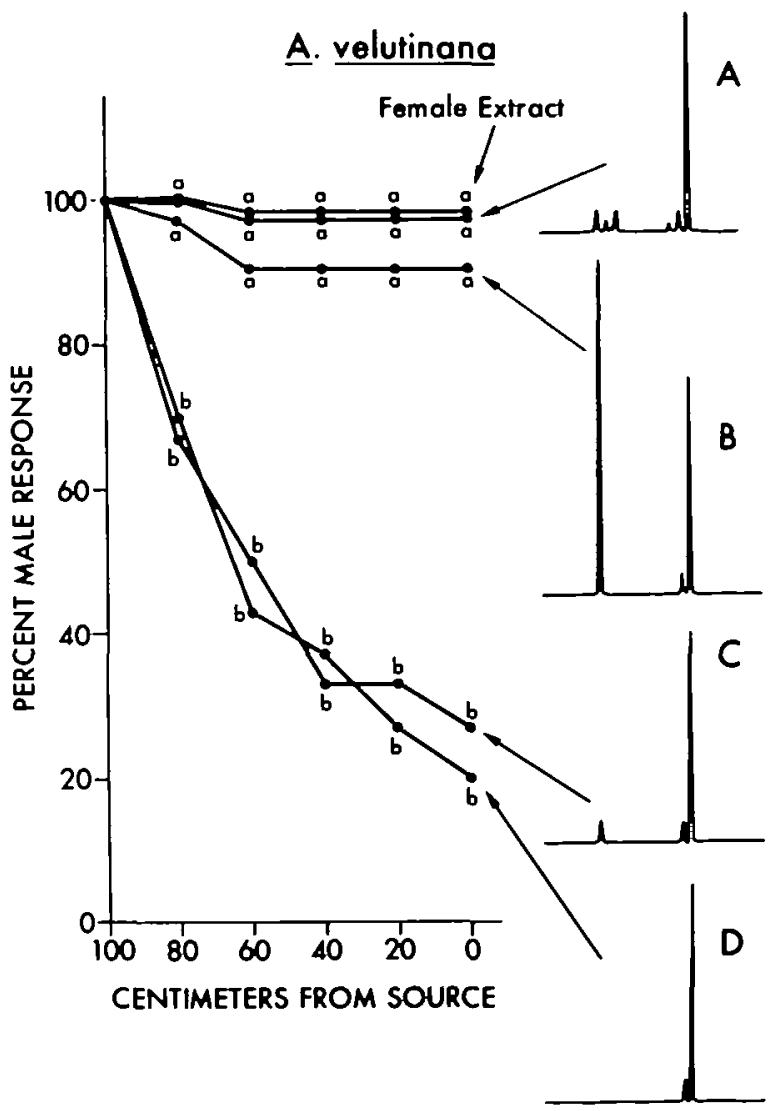

Fig. 4. Flight-tunnel responses of male redbanded leafroller moths to a female extract, to the seven-component female-produced blend ( $A$, the large peak in the trace is the major component, Z11-14:OAc), the threecomponent blend including excess dodecyl acetate identified from field trapping experiments (B), the same three-component mix including the proportion of dodecyl acetate found in females (C), and the two-component mix of Z- and E1 1-14:OAc (9\% E) lacking dodecyl acetate (D). Data from Bjostad et al. (1985).

\section{The blend paradigm and signal specificity: a case study with two noctuid moths}

As noted several times in this review, one of the fundamental themes in pheromone research is that multicomponent blends function as specific long-range mate recognition signals for males. We believe that our results and our conclusions concerning the importance of the female-released blend and its influence on the active space of the pheromone are in better agreement with this concept than the alternative argument emphasizing the functional role of the pheromone components. There are at least two major ways in which selection could favor male sensitivity to a unique blend of chemicals. First, on an intraspecific level, where male-male competition for available females is a potential selective force (Thornhill and Alcock, 1983) one could postulate that enhanced sensitivity to the pheromone blend will result in males being able to more 
rapidly locate conspecific females. Second, on an interspecific level, sensitivity to a specific blend or ratio of components could enable males to prevent making mistakes by being attracted to a closely related female.

That both types of selection pressure might be operative is suggested by the results of a comparative study between the cabbage looper and a closely related noctuid species, the soybean looper moth Pseudoplusia includens (Walker). These two species share a common major component, Z7-12:OAc, and two minor components, 12:OAc and 11-12:OAc. In addition the soybean looper pheromone contains Z7-12:propionate and Z7-12:butanoate, thus separating it as a distinct signal from the six-component cabbage looper blend (Linn et al., 1988b).

Flight tunnel and field studies with both species showed that, whereas cross-attraction did occur, it was quite low and appeared to be the result of two different processes. For the cabbage looper, males were attracted in significantly greater numbers to their conspecific blend than to Z7-12:OAc alone. In two of the three years in which field tests were conducted, male cabbage loopers were not even caught in traps baited with Z7-12:OAc alone or the soybean looper blend. A low level of cross-attraction was observed in one of the field tests as well as in the flight tunnel, but it was not significantly different from the level of attraction to Z7-12:OAc alone. This suggested that the low level of cross-attraction was the result of a low proportion of cabbage looper males responding only to the Z7-12:OAc component present in the soybean looper blend. These males represent a low proportion of the population that display low discriminatory capabilities. Thus, for the cabbage looper, a lower threshold for response to the blend compared with the major component alone appeared to mediate high levels of conspecific attraction and low levels of cross-attraction.

As for the soybean looper, male sensitivity to their conspecific blend was less obvious than with the cabbage looper, when compared with the response to Z7-12:OAc alone. Cross-attraction of male soybean loopers to the cabbage looper blend, however, was very low and flight tunnel studies indicated that this was the result of a very different process than observed for the cabbage looper. Male soybean loopers, in the presence of the cabbage looper blend, exhibited significant levels of arrested upwind flight, suggesting that they were responding to a compound in the cabbage looper pheromone that was acting as a behavioral antagonist. Independent electrophysiological studies by Grant et al. (1988) have supported this by showing that male soybean loopers have a highly specific receptor response for Z5-12:OAc, a component of the cabbage looper pheromone. Field studies further confirmed the interspecific action of this compound in that when it was added to the five-component soybean looper blend, the trap catch of male soybean loopers was significantly depressed (Grant et al., 1988).

The presence of behavioral antagonists in the communication systems of these two closely related species raises an important point concerning the action of pheromone components, and the criteria established to evaluate these chemicals behaviorally, i.e. that a compound need not necessarily enhance or elicit a specific behavior to be a valid component of the pheromone. One of the unresolved questions from the cabbage looper study, concerns the adaptiveness of the observed redundancy. If we assume that the released complement of components is the pheromone for a given species, then we should expect each component to impact on male behavior in some way. The observed redundancy, however, implies that some of the components released by female cabbage 
loopers are neutral in their effect. If this is indeed the case, we are forced to ask why females would continue to produce components that are unnecessary for attracting males. At the present time we can propose three possibilities. The first is that our assumption is wrong, and that some minor components are produced and released as a by-product of the synthesis. The neutral impact of these components would not deter from the importance of the active blend being essential for male response, nor would it necessitate a change in the experimental protocol described above. Second, if individual minor components were not essential for maximal response levels in male cabbage loopers, the fact that the minor components could substitute for one another only in very specific ways indicates that males are capable of detecting all of the minor components. This suggests the possibility that components might interact at common receptor sites, perhaps to amplify the signal in some manner. So little is known about the interaction of components, or blends of components, with antennal receptor sites that it is difficult to do more than speculate about this possible action. Third, what appear to be neutral components may in fact have important roles as interspecific signals, specifically as behavioral antagonists between closely related species. This is clearly the case with the soybean looper, and emphasizes the point that a compound may have a number of 'functions' in the communication system.

The action of interspecific behavioral antagonists is now documented for a number of species (see Linn et al., 1984; Glover et al., 1989) and we believe this phenomenon will be of increasing interest in the coming years. The activity of these chemicals represents an opportunity to explore several ideas in evolutionary theory, including their possible adaptive role as interspecific isolating mechanisms and examples of character displacement in the mating systems of closely related species.

\section{Summary}

The results of our studies on the functional role of pheromone components and the active space of the pheromone dramatically illustrate the importance of the interaction between chemical, biochemical and behavioral investigations. The success of these studies was clearly linked to a correct interpretation and understanding of the relevant stimulus affecting male response (see Bjostad, 1989). New evidence from biochemical studies stimulated a re-analysis of the cabbage looper pheromone, leading to the development of a new experimental approach to the analysis of male behavior, and subsequently to the reversal of an established paradigm. The new approach to behavioral analysis in turn has led to unexpected findings, e.g. the redundancy phenomenon, that have ultimately directed our investigations to the level of individuals interacting, both intraspecifically as well as interspecifically, at the population level.

\section{Conclusions}

Studies on the response specificity of male moths to their sex pheromone has progresesed from the investigatory stage to one of hypothesis testing and the formulation of concepts that have application not only to the study of insect chemical communication, but also to studies on olfaction in general. Central to the success of these investigations has been the increased application of sensitive analytical techniques, as well as close cooperation and dialogue between those involved in chemical, biochemical and 
behavioral studies. Increasingly, this dialogue has also extended to those involved in electrophysiological studies, so that there is at the present time a more integrated effort in studying these chemical communication systems (see Christensen et al., 1989).

\section{References}

Baker,T.C. (1985) Insect mating and courtship behaviour. In Kerkut,G.A. and Gilbert,L.I. (eds), Comprehensive Insect Physiology, Biochemistry, and Pharmacology. Pergamon Press, New York, Vol. 9, pp. $621-672$.

Baker,T.C. and Cardé,R.T. (1979) Analysis of pheromone-mediated flight behavior in male Grapholitha molesta, the Oriental fruit moth. Environ. Entomol., 8, 956-968.

Baker,T.C. and Linn,C.E., Jr (1984) Wind tunnels in pheromone research. In Hummel,H.E. and Miller,T.A. (eds), Techniques in Pheromone Research. Springer Verlag, New York, pp. 75-110.

Baker,T.C. and Roelofs,W.L. (1981) Initiation and termination of Oriental fruit moth male response to pheromone concentrations in the field. Environ. Entomol., 10, 211-218.

Baker,T.C., Cardé,R.T. and Roelofs, W.L. (1976) Behavioral responses of male Argyrotaenia velutinana to components of its sex pheromone. J. Chem. Ecol., 2, 333-352.

Baker, T.C., Meyer,W.R. and Roelofs, W.L. (1981) Sex pheromone dosage and blend specificity of response by Oriental frutt moths. Entomol. Exp. Appl., 30, 269-279.

Baker,T.C., Hansson,W.S., Löfstedt,C. and Löfqvist,J. (1989) Adaptation of male moth antennal neurons in a pheromone plume is associated with cessation of pheromone-mediated flight. Chem. Senses, 14, $439-448$.

Berger,R.S. (1966) Isolation, identification, and synthesis of the sex attractant of the cabbage looper, Trichoplusia ni. Ann. Entomol. Soc. Am., 59, 767-771.

Bjostad,L.B. (1989) Chemical characterization of sex pheromones and their biosynthetc intermediates. Chem. Senses, 14, 411-420.

Bjostad,L.B., Gaston,L.K., Noble,L.L., Moyer,J.H. and Shorey,H.H. (1980) Dodecyl acetate, a second pheromone component of the cabbage looper, Trichoplusia ni. J. Chem. Ecol., 6, 727-734.

Bjostad,L.B., Linn,C.E., Jr, Roelofs, W.L. and Du,J.-W. (1985) Identification of new pheromone components in Trichoplusia $n i$ and Argyrotainea velutinana, predicted from biosynthetic precursors. In Acree,T. and Soderlund,D. (eds), Semiochemistry: Flavors and Pheromones. Walter de Guyter \& Co., New York, pp. $223-238$.

Carde,A.M., Baker,T.C. and Cardé,R.T. (1979) Identification of a four-component sex pheromone of the female Oriental fruit moth, Grapholitha molesta (Lepidoptera: Tortricidae). J. Chem. Ecol., 5, 423-427.

Carde,R.T. (1979) Behavioral responses of moths to female-produced pheromones and the utilization of attractant-baited traps for population monitoring. In Rabb,R.L. and Kennedy,G.G. (eds), Movement of Highly Mobile Insects: Concepts and Methodology in Research. University Graphics, North Carolina State University, Raleigh, NC, pp. 286-315.

Carde,R.T. and Baker,T.C. (1984) Sexual communication in insects. In Bell,W. and Carde,R.T. (eds), Chemical Ecology of Insects. Sinauer Assoc., Sunderlund, MA, pp. 355-386.

Carde,R.T. and Charlton,R.E. (1984) Olfactory sexual communication in Lepidoptera: strategy, sensitivity and selectivity. In Lewis, T. (ed.), Insect Communication. Academic Press, New York, pp. 241-265.

CardE,R.T. and Webster,R. (1981) Endogenous and exogenous factors controlling insect sex pheromone production and responsiveness, particularly among the Lepidoptera. Scientific Papers of the Institute of Organic and Physical Chemistry of Wroclaw Technical University, no. 22, Conference 7, pp. 978-991.

Carde,R.T., Baker,T.C. and Roelofs,W.L. (1975a) Ethological function of components of a sex attractant system for Oriental fruit moth males, Grapholitha molesta. J. Chem. Ecol., 1, 475-491.

Carde,R.T., Baker,T.C. and Roelofs,W.L. (1975b) Behavioural role of individual components of a multichemical attractant system in the Oriental fruit moth. Nature, 253, 348-349.

Christensen, T.A., Mustaparta,H. and Hildebrand,G. (1989) Discrimination of sex pheromone blends in the olfactory system of the moth. Chem. Senses, 14, 463-477.

Farkas, S.R., Shorey,H.H. and Gaston,L.K. (1974) Sex pheromones of Lepidoptera. Influence of pheromone concentration and visual cues on aerial odor-trail following by males of Pectinophora gossypiella (Lepidoptera: Gelechiidae). Ann. Ent. Soc. Am., 67, 633-638.

Glover, T.J., Perez,N. and Roelofs, W.L. (1988) Comparative analysis of sex-pheromone-response antagonists in three races of European corn borer. J. Chem. Ecol., 15, 863-873. 
Grant,A.J., O'Connell,R.J. and Hammond,A.M. (1988) A comparative study of pheromone perception in two species of noctuid moths. $J$. Insect Behav., 1, 75-96.

Haynes,K.F., Gaston,L.K., Mistrot Pope,M. and Baker,T.C. (1984) Potential for evolution of resistance to pheromones: interindividual and interpopulational variation in chemical communication system of pink bollworm moths. J. Chem. Ecol., 10, 1551-1565.

Howse,P.E., Lisk,J.C. and Bradshaw,J.W.S. (1986) The role of pheromones in the control of behavioural sequences in insects. In Payne,T.L., Birch,M.C. and Kennedy,C.E.J. (eds), Mechanisms in Insect Olfaction. Clarendon Press, Oxford, pp. 157-162.

Levine,M.W. and Shefner,J.M. (1981) Fundamentals of Sensation and Perception. Addison-Wesley, Reading, MA.

Linn,C.E., Jr and Gaston,L.K. (1981) Behavioural responses of male Trichoplusia $n$ in a sustained-flight tunnel to the two sex pheromone components. Environ. Entomol., 10, 379-385.

Linn,C.E., Jr and Roelofs, W.L. (1981) Modification of sex pheromone blend discrimination in male Oriental fruit moths by pre-exposure to (E)-8-dodecenyl acetate. Physiol. Entomol., 6, 421-429.

Linn,C.E., Jr and Roelofs, W.L. (1983) Effect of varying proportions of the alcohol component on sex pheromone blend discrimintion in male Oriental fruit moths. Physiol. Entomol., 8, 291-306.

Linn,C.E., Jr and Roelofs, W.L. (1985) Response specificity of male pink bollworm moths to different blends and dosages of pheromone. J. Chem. Ecol., 11, 1583-1590.

Linn,C.E., Jr, Bjostad,L.B., Du,J.-W. and Roelofs, W.L. (1984) Redundancy in a chemical signal: behavioral responses of male Trichoplusia ni to a 6-component sex pheromone blend. J. Chem. Ecol., 10, 1635-1658.

Linn,C.E., Jr, Campbell,M.G. and Roelofs, W.L. (1985) Male moth sensitivity to multicomponent pheromones: the critical role of the female-released blend in determining the functional role of components and the active space of the pheromone. $J$. Chem. Ecol., 12, 659-668.

Linn,C.E., Jr, Campbell,M.G. and Roelofs,W.L. (1987) Pheromone components and active spaces: what do male moths smell and where do they smell it? Science, 237, 650-652.

Linn,C.E., Jr, Campbell,M.G. and Roelofs,W.L. (1988a) Temperature modulation of behavioral thresholds cotrolling male moth sex pheromone response specificity. Physiol. Entomol., 13, 59-67.

Linn,C.E., Jr, Hammond,A., Du,J.-W. and Roelofs, W.L. (1988b) Specificity of male response to multicomponent pheromones in noctuid moths Trichoplusia ni and Pseudoplusia includens. J. Chem. Ecol., $14,47-57$.

Roelofs,W.L. (1978) Threshold hypothesis for pheromone perception. J. Chem. Ecol., 4, 685-699.

Roelofs, W.L. and Bjostad,L.B. (1984) Biosynthesis of lepidopteran pheromones. Bioorg. Chem, 12, $279-298$.

Roelofs, W.L. and Brown,R.L. (1982) Pheromones and evolutionary relationships of Tortricidae. Annu. Rev. Ecol. Syst., 13, 395-422.

Roelofs, W.L. and Carde,R.T. (1977) Responses of Lepidoptera to synthetic sex pheromone chemicals and their analogues. Annu. Rev. Entomol., 22, 377-405.

Shorey,H.H. (1976) Animal Communication by Pheromones. Academic Press, New York.

Tamaki,Y. (1985) Sex pheromones. In Kerkut,G. and Gilbert,L. (eds), Insect Physiology, Biochemistry, and Pharmacology. Pergamon Press, New York, Vol. 9, pp. 145-192.

Thomhill,R. and Alcock,J. (1983) The Evolution of Insect Mating Systems. Harvard University Press, Cambridge, MA, p. 53.

Received on August 8, 1988; accepted on January 1, 1989 